\title{
Traffic Prediction for Agent Route Planning
}

\author{
Jan D. Gehrke ${ }^{1}$ and Janusz Wojtusiak ${ }^{2}$ \\ ${ }^{1}$ Center for Computing Technologies (TZI) \\ University of Bremen, 28359 Bremen, Germany \\ jgehrke@tzi.de \\ ${ }^{2}$ Machine Learning and Inference Laboratory \\ George Mason University, Fairfax, VA 22030, USA \\ jwojt@mli.gmu.edu
}

\begin{abstract}
This paper describes a methodology and initial results of predicting traffic by autonomous agents within a vehicle route planning system. The traffic predictions are made using AQ21, a natural induction system that learns and applies attributional rules. The presented methodology is implemented and experimentally evaluated within a multiagent-based simulation system. Initial results obtained by simulation indicate advantage of agents using AQ21 predictions when compared to naïve agents that make no predictions and agents that use only weather-related information.
\end{abstract}

Keywords:Traffic Prediction, Intelligent Agents, Natural Induction.

\section{Introduction}

The importance of information technology (IT) in logistics has increased remarkably. IT systems support or take responsibility for logistics planning of a forwarder's whole motor pool or single vehicles. The just-in-time paradigm demands for high robustness to situation changes and real-time coordination abilities of all participants in the logistics network. These requirements are facilitated by pervasive mobile communication networks and devices as well as intelligent systems processing incoming information [1]. Due to the fierce competition in the logistics service market, companies are searching for new technologies that advance quality of service (e.g., promptitude and robustness to disturbances) and cost of carriage. Thus, even seemingly small differences may bring about a considerable competitive advantage and high economic impact.

The agent-based approach to intelligent logistics systems delegates the planning and decision making from central planning systems to single logistic entities, such as trucks and containers, that decide autonomously and locally. With reduced complexity for (re-)planning, this decentralized approach aims at increased robustness to changes such as new transport orders or vehicle breakdown. Approaches for agent-based transport planning and scheduling have been proposed by, e.g., Dorer and Callisti [2] and Bürckert et al. [3].

The ability of autonomous systems to react to situation changes calls for situation awareness provided by sensors, other agents, or external sources such as 
databases. To make use of this information the agent needs to know its relevance, i.e., how information will influence its cost or utility function during planning of actions. Furthermore, the agent has to consider the spatio-temporal scope where some information is valuable. Because the scope of agent planning is the near future, agents also need prediction abilities.

In this paper we examine the influence of environmental knowledge in vehicle route planning in simulation experiments. In particular, we examine how road traffic predictions used in route planning affect vehicles' performance measured as the time needed to reach their destination. In order to do this, we compare performance of ignorant agents, with agents that are provided with weather information and agents that use rules induced from historical traffic data for predictions. The rules used in the latter approach are learned using the natural induction system AQ21.

\section{Situation-Aware Vehicle Route Planning}

The performance of an intelligent planning systems depends on a proper analysis of the current situation and prediction of the future. Depending on the domain, the planning scope may cover a few hours for regional transports or some days for international or overseas routes. Thus, an important requirement for intelligent planning systems is situation awareness [4 as well as situation prediction.

In order to investigate the impact of such abilities in logistics and to understand the spatial and temporal constraints we reduce the problem to a simplified single vehicle transportation scenario. An agent represents a truck that aims at finding the fastest route in a graph-based highway grid. This becomes a complex problem because there are highly dynamic environmental conditions that may enforce or reduce the speed at which the vehicle may travel. The examined conditions include traffic density and weather that vary in space and time.

In this study, the vehicle route planning applies an $A^{*}$ search algorithm with cost function (1) for reaching destination $d$ when using (partial) route $r$ at departure time $t_{\mathrm{dep}}$ :

$$
f\left(r, d, t_{\mathrm{dep}}\right)=g\left(r, t_{\mathrm{dep}}\right)+h\left(e n d_{r}, d\right)
$$

with $g$ as the estimated driving time for $r$ and $h$ as the estimated driving time from $r$ 's endpoint $e n d_{r}$ to $d$. Heuristics $h$ is calculated as driving time at straight line distance from $e n d_{r}$ to $d$ at maximum vehicle speed. The route $r$ consists of $n$ consecutive edges (i.e., roads) $e_{i} \in r$ with $0 \leq i<n$. The route segment of first $k$ edges is denoted by $r_{k-1}$. The driving time $g$ on route $r$ is calculated by:

$$
g\left(r, t_{\mathrm{dep}}\right)=\sum_{i=0}^{n-1} \frac{\text { length }\left(e_{i}\right)}{v_{\mathrm{est}}\left(e_{i}, t_{\mathrm{dep}, e_{i}}\right)}
$$

with $v_{\text {est }}$ as the estimated vehicle speed on an edge which depends on the vehicle agent implementation (Sect. 31). Edge departure time for $i>0$ is defined by

$$
t_{\mathrm{dep}, e_{i}}=g\left(r_{i-1}, t_{\mathrm{dep}}\right)
$$


Because this setting ensures the criteria for the $\mathrm{A}^{*}$ algorithm (non-negative costs and optimistic heuristics) it guarantees the optimal solution. However, the found route is optimal only provided that knowledge about the environment used in the cost function is complete and correct. But assumptions on future road conditions are possibly wrong because the environment continuously changes in a way that cannot be precisely predicted.

\section{Simulation Model}

In order to evaluate the impact of environmental knowledge and driving time prediction abilities we set up experiments with the multiagent-based simulation system PlaSMA1. The system applies discrete event, distributed simulation using software agents as logical simulation processes. Thus, PlaSMA provides a natural way to evaluate software agents behavior and interactions.

For the purpose of vehicle route planning, the simulation model includes two world agents and multiple vehicle agents. The world agents simulate weather and traffic within the simulation environment. The vehicle agents can be categorized in three classes: the ignorant agent, the weather-aware agent, and the predictive agent. While all vehicle agents use $\mathrm{A}^{*}$ search with time cost function for routing, they use different knowledge in planning and thereby provide a comparative measure to evaluate the impact of their knowledge.

\subsection{Weather and Traffic}

For the logistics scenario we set up a road network as a $6 \times 6$ grid graph with a set $\mathcal{E}$ of directed edges (i.e., unidirectional roads) of $100 \mathrm{~km}$ length each. This idealized grid structure ensures that results are not biased by a special network structure that may have no implications for the general problem. Nevertheless, experiments can be conducted with other network structures as well. The two world agents generate simulation events that affect this graph with respect to traffic density dens $(e, t)$ and maximum safe speed $v_{\text {safe }}(e, t)$ for each edge $e \in \mathcal{E}$ at simulation time $t$. dens $(e, t)$ is a linear traffic quality value that is normalized to 1 and indicates the ability of a vehicle to drive at a reference speed $v_{\text {ref }}$, i. e., maximum speed is

$$
v_{\max }(e, t)=v_{\text {ref }} \cdot \operatorname{dens}(e, t)
$$

For our experiments we use $v_{\text {ref }}=130 \mathrm{~km} / \mathrm{h}$ for all roads. This value corresponds to the recommended speed on German Autobahn roads for passenger cars.

Speed $v_{\text {safe }}$ depends on the current weather on each road. Different roads may have different weather but the weather within the area of each road is homogeneous. The simulation model is designed for a set of qualitative weather types $\mathcal{W}=\{$ VeryBad,Bad, Moderate, Good $\}$. weather $(e, t)$ describes the weather at an edge $e$ at time $t$. Each weather type $w \in \mathcal{W}$ corresponds to a truck-oriented maximum safe speed $v_{\text {safe }}(w)$ when facing that weather:

$$
\begin{gathered}
v_{\text {safe }}(\text { VeryBad })=35 \mathrm{~km} / \mathrm{h}, v_{\text {safe }}(\text { Bad })=65 \mathrm{~km} / \mathrm{h} \\
v_{\text {safe }}(\text { Moderate })=80 \mathrm{~km} / \mathrm{h}, v_{\text {safe }}(\text { Good })=100 \mathrm{~km} / \mathrm{h}
\end{gathered}
$$

\footnotetext{
${ }^{1}$ Available from http://plasma.informatik.uni-bremen.de/
} 
Then, the maximum safe speed for each edge $e \in \mathcal{E}$ at time $t$ is defined by

$$
v_{\text {safe }}(e, t)=v_{\text {safe }}(\text { weather }(e, t))
$$

The minimum of $v_{\max }(e, t)$ and $v_{\text {safe }}(\mathrm{e}, \mathrm{t})$ determines the maximum possible average speed $v_{\text {avg }}(e, t)$ of a vehicle on edge $e$.

Weather Generation. The weather agent updates weather in the interval $\Delta t_{\mathcal{W}}$ separately for each edge. The new weather type $w \in \mathcal{W}$ on each edge $e$ depends on the previous weather at $e$ and the basic probability distribution $\mathbf{P}(\mathcal{W})$ for weather which is assumed to be location-independent.

The next weather is determined by the weather $w$ randomly drawn from $\mathcal{W}$ according to $\mathbf{P}(\mathcal{W})$ but changes may be constrained depending on current weather to avoid sudden changes as determined by transition probability model $\mathbf{P}\left(\mathcal{W}_{t+\Delta t_{\mathcal{W}}} \mid \mathcal{W}_{t}\right)$. The actual distributions for weather and length of $\Delta t_{\mathcal{W}}$ are subject of the experimental setup (Sect. 6).

Traffic Generation. Similarly to the weather agent, the traffic simulation agent updates traffic density on each edge in interval $\Delta t_{\mathcal{T}}$. Though the generated traffic density is a real number, the traffic generator is based on a qualitative traffic model with a set of traffic classes $\mathcal{T}$ covering disjoint intervals of traffic density. Similarly to levels of service A to F in US Highway Capacity Manual [5], the model distinguishes six traffic classes $\mathcal{T}=\{$ VeryLow, Low, Medium, High, VeryHigh, Jam $\}$ and the following traffic density intervals:

$$
\begin{gathered}
\text { VeryLow }=[0.0,0.1), \text { Low }=[0.1,0.25), \text { Medium }=[0.25,0.4) \\
\text { High }=[0.4,0.6), \text { VeryHigh }=[0.6,0.85), \text { and Jam }=[0.85,1.0]
\end{gathered}
$$

$\mathcal{T}_{\mu}$ denotes the mean value of a traffic class. The traffic density on an edge is determined by an edge-specific density matrix for time of day and day of week. For this purpose, we analyzed traffic volume data from German (BASt) and Austrian (ASFINAG) agencies that count traffic on national highways. According to the combined days and hours in aggregated agency models we set up the basic model matrix depicted in Table 1 that determines the basic mean traffic density value $\mathcal{T}_{\mu}(t)$. The basic model is used for all edges but each edge $e$ has an additional and time-independent traffic bias function $\Delta$ dens $(e)$ that shifts the basic model to higher or lower traffic densities.

The actual values for the density bias function $\Delta$ dens depend on the experimental setup (see Sect. 6). The traffic generation world agent calculates the density dens $(e, t)$ with Gaussian distribution $N(\mu, \sigma)$ :

$$
\operatorname{dens}(e, t) \sim N\left(\mathcal{T}_{\mu}(t)+\Delta \operatorname{dens}(e), \sigma\right)
$$

\subsection{Vehicle Agents}

There are three kinds of vehicle agents. The ignorant agent is not aware of any environmental information and has no predictive abilities. Like all other vehicle 
Table 1. Basic traffic density matrix

\begin{tabular}{|c|c|c|c|c|c|}
\hline & Mo & Tu-Th & Fr & Sa & Su \\
\hline \hline Morning, 6am-10am & High & Medium & Medium & VeryLow & VeryLow \\
\hline Noon, 10am-3pm & Low & Low & High & Low & Low \\
\hline Afternoon, 3pm-7pm & Medium & Medium & High & Medium & Medium \\
\hline Evening, 7pm-10pm & Low & Low & Medium & Low & Low \\
\hline Night, 10pm-6am & VeryLow & VeryLow & Low & VeryLow & Low \\
\hline
\end{tabular}

agents it uses the algorithm described in Sect.2 Because this agent assumes that all roads allow equal speed its dominant planning criterion is distance. Within the road grid the ignorant agent chooses a route that most closely matches the straight line, disregarding possible bad weather or traffic conditions.

The weather-aware agent is a vehicle agent that acquires status information on weather for relevant locations. The agent uses this information to determine speed $v_{\text {est }}\left(e, t_{\mathrm{dep}, e}\right)$ for route planning (Sect. 2). It does not attempt to predict any traffic or changes of weather but naïvely assumes that there is no (relevant) traffic or change of weather w.r.t current time $t_{\text {cur }}$, i. e.

$$
v_{\mathrm{est}}\left(e, t_{\mathrm{dep}, e}\right)=v_{\mathrm{safe}}\left(e, t_{\mathrm{cur}}\right)=v_{\mathrm{safe}}\left(\text { weather }\left(e, t_{\mathrm{cur}}\right)\right)
$$

The status information is provided by the weather agent. Which locations are considered interesting depends on the current vehicle location, its destination, and a lookahead parameter $\lambda . \lambda$ specifies the spatial range and the area for which the agent acquires environmental status information. The actual lookahead distance used in this study is $\lambda \cdot 100 \mathrm{~km}$. Because the agent assumes the weather to be static when planning the fastest route, it may turn out to be wrong when reaching edges that are far ahead. However, vehicle agents may reconsider their route at each junction, i.e., wrong decisions can be corrected if there are new, better alternatives.

The predictive agent is an extension of the weather-aware agent. It also assumes that weather at edge $e$ will not change until arrival at $e$. But it also predicts traffic at $e$ for $t_{\mathrm{dep}, e}$ to determine $v_{\mathrm{est}}\left(e, t_{\mathrm{dep}, e}\right)$ (Sect. 2). The predictions rely on previous experiences that are used as data to learn situation- and edge-specific rules for expected speed (Sect. 5). Besides time of day and day of week these predictions may depend on weather and thus on $\lambda$, too. Edges that are not within $\lambda$ distance are assumed to have the most likely weather, e.g. Moderate.

\section{Natural Induction}

In contrast to most methods known in the literature, including different forms of statistical learning, the approach used in this study puts an equal importance to accuracy and interpretability of learned models. While the importance of the former does not require any justification, the latter may not be clear, especially 
in the area in which learned knowledge is used by autonomous agents. Models learned by many methods can be regarded as a "black box" which may give very good predictions, but it is hard to understand and often impossible to validate or refine by human experts. Models described in a language that is easy to understand, for example, natural language or easy to interpret rules, can be modified by experts to reflect their background knowledge and improve predictions. In this study we use natural induction [6] that is an approach to inductive learning whose goal is to achieve high understandability of derived knowledge. It uses a highly expressive language attributional calculus (AC) that combines predicate, propositional and multi-valued logics. Because all forms of knowledge used in AC corresponds to different constructs in natural language, its expressions are easy to understand.

\subsection{Knowledge Representation}

The main form of knowledge in $\mathrm{AC}$ is an attributional rule. Attributional rules, which follow the general if ...then ... schema, are more general than those learned by most learning programs. This is because attributional rules use more expressive language which allows creating simpler descriptions than normal rules. A basic form of attributional rules is (8) .

$$
\text { CONSEQUENT } \Leftarrow \mathrm{PREMISE}
$$

Here, CONSEQUENT and PREMISE are conjunctions of attributional conditions. In this study we used attributional conditions in the form $[L \operatorname{rel} R]$ where $L$ is an attribute; $R$ is a value, a disjunction of values, or a conjunction of values if $L$ is a compound attribute; and rel is a relation that applies to $L$ and $R$. Other forms of attributional conditions may involve count attributes, simple arithmetical expressions, conjunctions and disjunctions of attributes, comparison or attributes, etc. 7 .

\subsection{The AQ21 Machine Learning System}

A simple form of natural induction is implemented in the currently developed AQ21 system [7. Given input data, problem definition, and optional background knowledge, AQ21 induces rules in the form (8), or in more advanced forms, describing one or more class in the data. A set of rules constituting a description of a given class is called a ruleset. By repeating learning for all classes defined by values of an output attribute, AQ21 generates a classifier.

In order to learn rules for a given class AQ21 starts with one example, called a seed, belonging to the class. It generates a star, which is a set of maximally general rules that cover the seed and do not cover any examples from other classes. This is done by repeating an extension-against operation that generalizes the seed against examples not belonging to the concept being learned. Results of applying the extension-against are intersected and the best rules are selected according to user-defined criteria. If selected rules do not cover all examples belonging to the class, another seed is selected (from the not covered examples) 
and additional rules are learned. The process is repeated until all examples of the class are covered by the learned rules. AQ21 implements several modifications to the above basic algorithm as described, for example, in [7].

\section{Learning and Applying Traffic Models}

In the presented study traffic models were learned using simulated data collected over 15 years of simulation time. The training data consisted of 131,488 examples for each type of edge. To learn traffic models we used the AQ21 system (Sect.4.2). It was executed with different settings of parameters, from which we selected the best according to predictive accuracy on testing data and simulation results. We applied the program in two modes, theory formation (TF) and approximate theory formation (ATF). The TF mode learns complete and consistent rulesets w.r.t. the training data, while the ATF mode allows partial inconsistence and incompleteness (e.g. in the presence of noise) by optimizing the $Q(w)$ quality measure [8]. In this study the weight, w, of completeness against consistency gain was 0.1 .

Many training examples are ambiguous, meaning that for identical values of Day, Time, and Weather, different values of Speed are assigned. Among several methods for solving ambiguity available in AQ21 we investigated two. The first method, here called majority, assigned the most frequent class to all ambiguous examples, and the second method, here called pos, always treats ambiguous examples as positive examples. Details of the methods are in [9].

Application of learned models to classify new examples is done by executing AQ21's testing module. The program is provided with input files consisting of a testing problem description, learned models and one or more testing examples. In the presented experiments we tested two methods of evaluating rules, namely strict in which an example either matches a rule or not, and flexible in which the program calculates the degree to which examples match rules. In the latter case the degree of match equals the number of matching conditions in the rule to the total number of conditions in the rule. Detailed description of the parameters and other rule matching schemas are in 69. Two methods of resolving imprecise classifications are tested here. The first method is pessimistic, namely it assumes the worst of the given answers (the lowest predicted speed). The second method is based on frequency of classes. For instance, if an example matches two classes "slow" and "very slow," and the former is more frequent in the training data, it is reasonable to assume that the prediction should be "slow."

Average predictive accuracies and precisions 9] of models learned and applied with different AQ21 parameters on testing data with variances 0.001 and 0.01 for dens $(e, t)$ ranged from $91 \%$ to $100 \%$ and $71 \%$ to $100 \%$, respectively. The best results were obtained by AQ21 in the theory formation mode, with ambiguities treated as majority and flexible rule interpretation. There were $95 \%$ predictive accuracy, and $100 \%$ precision. All presented values are averaged for all types of roads in the simulation model. As an example, the following rule has been learned using the TF mode. It predicts that expected maximum speed for a 
given road is $60 \mathrm{kmph}$ on Monday mornings provided moderate to good weather conditions.

[Speed=SPEED_60]

$<=[$ Day=Mo] \& [Time=morning] \& [Weather=moderate..good]

\section{Experimental Evaluation}

For evaluation we conducted experiments using the simulation system PlaSMA. Each experiment is specified by a simulation model parameter setting and 9 participating vehicle agents. There is one ignorant (named "IA"), one weather-aware ("WA"), and seven traffic-predicting agents ("AQ"). The latter are differing in the applied prediction rulesets for each edge (Sect. 51) as indicated by their index.

The agent named $A Q_{M}$ is a traffic-predicting agent whose rules have been created manually knowing the actual traffic simulation model. Thus, this agent should provide results close to the achievable optimum for the applied algorithm. All agents try to optimize the driving time of a $1000 \mathrm{~km}$ trip in the road network. Simulation results are provided as an average driving time and its standard deviation for each vehicle agent and parameter setting. For statistical significance experiments were repeated between 4200 and 4800 times with each run corresponding to one trip. With significance level $\alpha=0.05$ stated average values do not differ more than 0.02 hours from the actual value.

Most of the model parameters have been examined in prior studies [10]. For all experiments presented in this paper we set parameters to $\Delta t_{\mathcal{T}}=1 \mathrm{~h}, \Delta t_{\mathcal{W}}=3 \mathrm{~h}$, and $\lambda=2$. Weather probabilities are set to

$$
\begin{aligned}
\mathbf{P}(\mathcal{W})= & \{P(\mathcal{W}=\text { VeryBad })=0.05, P(\mathcal{W}=\text { Bad })=0.2, \\
& P(\mathcal{W}=\text { Moderate })=0.55, P(\mathcal{W}=\text { Good })=0.2\}
\end{aligned}
$$

The edge-specific density bias $\Delta$ dens $(e)$ was examined in a setting that is characterized by a fairly well-shuffled distribution of slower and faster edges (see Fig. 1). As an exception, the grid also includes three edges (dashed line) that form a fast partial route. These edges are not part of routes that most closely match the theoretical straight line route. Hence, ignorant vehicles that are dominated by the straight line heuristics and always choose such centered routes will not be affected. Weather-aware agents should not benefit as much as traffic predicting agents because only the latter will actually realize the traffic properties and consider them in planning.

Table 2 shows the simulation results for traffic density setting depicted in Fig. 1. With variance $\sigma_{\mathcal{T}}^{2}=0.001$, the ignorant agent needs $13.31 \pm 0.87$ hours driving time on average. The weather-aware agent needs $12.61 \pm 0.78 \mathrm{~h}$, hence, it is $0.7 \mathrm{~h}$ or $5.3 \%$ faster than the ignorant agent. The prediction ruleset $A Q_{3}$ (AQ21 mode: TF, majority, strict) performs best with $12.47 \pm 0.60 \mathrm{~h}(0.84 \mathrm{~h}$, $6.3 \%$ faster). The standard deviation is significantly lower than that of $I A$ and $W A$, too. $A Q_{3}$ and $A Q_{4}$ even come close to the reference ruleset $A Q_{M}$. With 


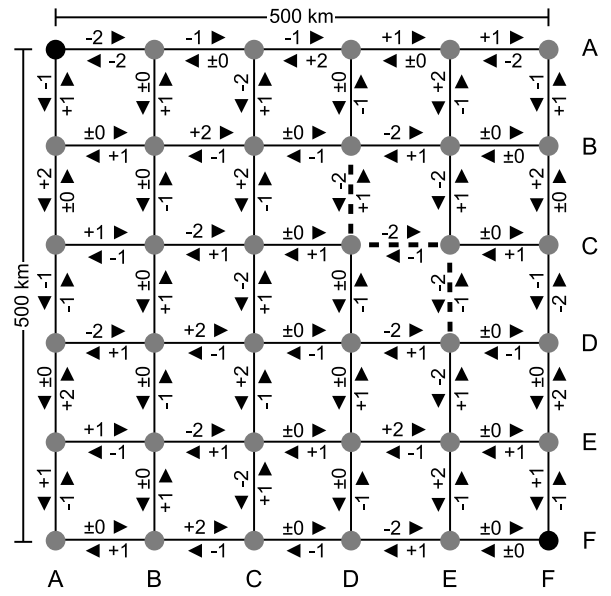

Fig. 1. Road grid annotated with $\Delta$ dens $(e) \cdot 10$

$\sigma_{\mathcal{T}}^{2}=0.01, A Q_{3}$ needs 0.16 hours longer due to more wrong predictions and all agents using TF mode perform equally good. The changes are rather small and all situation-aware agents still clearly outperform the ignorant agent. Thus, these agents can be considered sufficiently robust to less predictable environments.

Table 2. Average vehicle driving time in hours

\begin{tabular}{|c|c|c|c|c|c|c|c|c|c|c|c|}
\hline \multirow[t]{2}{*}{$\sigma_{\mathcal{T}}^{2}$} & \multirow[t]{2}{*}{ IA } & \multirow[t]{2}{*}{ WA } & \multirow[t]{2}{*}{$A Q_{M}$} & $\begin{array}{c}A Q_{1} \\
\mathrm{TF}\end{array}$ & $\begin{array}{c}A Q_{2} \\
\mathrm{TF}\end{array}$ & $\begin{array}{c}A Q_{3} \\
\mathrm{TF}\end{array}$ & $\begin{array}{c}A Q_{4} \\
\mathrm{TF}\end{array}$ & $\begin{array}{l}A Q_{5} \\
\mathrm{ATF}\end{array}$ & $\begin{array}{l}A Q_{6} \\
\text { ATF }\end{array}$ & $\begin{array}{l}A Q_{7} \\
\mathrm{ATF}\end{array}$ & $\begin{array}{l}A Q_{8} \\
\text { ATF }\end{array}$ \\
\hline & & & & pos,str & pos,flx & maj,str & $\mathrm{maj}, \mathrm{flx}$ & os,str & os, flx & aj,s & haj,f \\
\hline 0.001 & 31 & 61 & .49 & 12.51 & 12.56 & 12.47 & 12.50 & 12.62 & 12.63 & 12.54 & 12.57 \\
\hline & 1 & 0.78 & \pm 0.60 & \pm 0.64 & \pm 0.63 & \pm 0.60 & \pm 0.61 & \pm 0.60 & \pm 0.64 & \pm 0.64 & \pm 0.65 \\
\hline 0.01 & 13.44 & 12.74 & 12.64 & 12.63 & 12.63 & \begin{tabular}{|l|}
12.63 \\
\end{tabular} & 12.63 & 12.75 & 12.74 & 12.64 & 12.69 \\
\hline & \pm 0.9 & \pm 0.86 & \pm 0.6 & \pm 0.6 & \pm 0.6 & \pm 0.66 & \pm 0.66 & \pm 0.67 & \pm 0.64 & \pm 0.66 & \pm 0.71 \\
\hline
\end{tabular}

\section{Conclusions}

This paper presented an approach to the problem of predicting traffic for vehicle routing. Autonomous agents make predictions based on rules induced by the AQ21 system from historic data. By inducing attributional rules AQ21 realizes natural induction, that is an inductive learning process whose results are both accurate and easy to understand by people. Experiments performed within the PlaSMA multiagent-based simulation system indicated advantage of agents that use AQ21 predictions over naïve agents that consider only distance to the destination and agents that use only weather-related information.

Future research includes the comparison with different learning methods, investigation of the effect of the amount of historic data on the learning results 
and predictions, and investigation of the effects of changes of the environment on the predictions. Other important research directions include learning individualized models for each agent, based on the agent's experience and preferences, and application of the system in a real, not simulated, environment.

Acknowledgments. The authors thank Abdur Chowdhury for his contributions to experiment design and Jarek Pietrzykowski for his comments that helped to improve this paper. This research was partially funded by the German Research Foundation (DFG) within Collaborative Research Centre 637 (SFB 637). Development of the AQ21 system was partially funded by the National Science Foundation Grants IIS 9906858 and IIS 0097476.

\section{References}

1. Scholz-Reiter, B., Windt, K., Freitag, M.: Autonomous logistic processes: New demands and first approaches. In: Monostri, L. (ed.) Proceedings of the 37th CIRP International Seminar on Manufacturing Systems, pp. 357-362 (2004)

2. Dorer, K., Calisti, M.: An adaptive solution to dynamic transport optimization. In: Proceedings of AAMAS 2005, pp. 45-51 (2005)

3. Bürckert, H., Fischer, K., Vierke, G.: Holonic transport scheduling with teletruck. Journal of Applied Artificial Intelligence 14, 697-725 (2000)

4. Endsley, M.R.: Theoretical Underpinnings of Situation Awareness: A Critical Review. In: Endsley, M.R., Garland, D.J. (eds.) Situation Awareness, Analysis and Measurement, Lawrence Erlbaum Assoc., Mahwah (2000)

5. Transportation Research Board: Highway Capacity Manual (HCM) (2000)

6. Michalski, R.S.: Attributional Calculus: A Logic and Representation Language for Natural Induction. Tech. Report MLI 04-2, MLI, George Mason University (2004)

7. Wojtusiak, J., Michalski, R.S., Kaufman, K., Pietrzykowski, J.: The AQ21 Natural Induction program for pattern discovery: Initial version and its novel features. In: Proceedings of the 18th IEEE International Conference on Tools with Artificial Intelligence, Washington, DC (2006)

8. Kaufman, K., Michalski, R.S.: An Adjustable Rule Learner for Pattern Discovery Using the AQ Methodology. Journal of Intelligent Information Systems 14, 199-216 (2000)

9. Wojtusiak, J.: AQ21 User's Guide. Tech. Report MLI 04-3, MLI, George Mason University (2004) (updated, September 2005)

10. Gehrke, J.D., Wojtusiak, J.: A natural induction approach to traffic prediction for autonomous agent-based vehicle route planning. Tech. Report MLI 08-1, MLI, George Mason University (2008) 